\title{
ANALYSIS \\ Renal transplantation between HIV-positive donors and recipients justified
}

\author{
Elmi Muller, Zunaid Barday, Marc Mendelson, Delawir Kahn
}

HIV infection was previously an absolute contraindication to renal transplantation. However, with the advent of highly active antiretroviral therapy (HAART), renal transplantation using HIVnegative donor kidneys has successfully been employed for HIVinfected patients with end-stage renal failure. In resource-limited countries, places on dialysis programmes are severely restricted; HIV-infected patients, like many others with co-morbidity, are often denied treatment. Kidneys (and other organs) from HIVinfected deceased donors are discarded. The transplantation of
HIV-positive donor kidneys to HIV-infected recipients is now a viable alternative to chronic dialysis or transplantation of HIVnegative donor kidneys. This significantly increases the pool of donor kidneys to the advantage of HIV-positive and -negative patients. Arguments are presented that led to our initiation of renal transplantation from HIV-positive deceased donors to HIVpositive recipients at Groote Schuur Hospital, Cape Town.

S Afr Med J 2012;102(6):497-498.

\section{HIV-associated nephropathy - an increasing clinical problem}

While highly active antiretroviral therapy (HAART) has reduced the morbidity and mortality of HIV-infected people, HIV-associated chronic diseases place an increasingly heavy burden on health systems in South Africa (SA). HIV-associated nephropathy (HIVAN) is the leading cause of end-stage renal failure (ESRF) in HIV-infected patients in SA. ${ }^{1,2}$ In the USA, it is the fourth most common cause of ESRF among 20 - 64-year-old HIV-infected black patients (after diabetes, hypertension and chronic glomerulonephritis). ${ }^{3}$

A study at Chris Hani Baragwanath Hospital in Johannesburg demonstrated HIVAN in $27 \%$ of 455 HIV patients, ${ }^{4}$ while a study in Durban found HIVAN in $83 \%$ of cases of ESRF in antiretroviralnaive HIV-infected patients. ${ }^{2}$ Based on approximately 5.5 million people living with HIV/AIDS in SA, an estimated $10 \%$ will develop ESRF, despite standard antiretroviral therapy (ART). Some 550000 individuals may be expected to require renal replacement therapy (RRT) comprising dialysis and transplantation, considerably more than the SA population currently receiving dialysis, and exceeding the health service renal replacement capacity. Compounding this potentially massive increase in RRT candidates, is the shortage of deceased donor organs; at Groote Schuur Hospital (GSH) the number of deceased donor referrals has halved in the past 10 years.

\section{Criteria for renal replacement therapy in low- to middle-income countries}

A lack of dialysis slots exists worldwide. Emerging economies such as SA experience the greatest pressure of competition for haemo- and peritoneal dialysis slots. ${ }^{5}$ GSH has facilities for only

Dr E Muller and Professor D Kahn are both transplant surgeons at Groote Schuur Hospital and have an active interest in the transplantation of HIV-positive patients. Dr Z Barday is a transplant nephrologist at Groote Schuur Hospital and Professor M Mendelson is a specialist in infectious disease and HIV medicine at the same hospital.
100 haemodialysis and 50 peritoneal dialysis patients in its chronic dialysis programme at any one time, with concomitant pressure to transplant patients and free dialysis space. A strategy has been adopted to offer RRT to the healthiest and the young. As in other centres in SA, prospective patients are presented at weekly multidisciplinary assessment meetings to decide whether they are suitable for renal replacement. ${ }^{6}$ Criteria, including fitness to undergo renal transplantation, age, co-morbidity, social circumstances and psychological state, are strictly applied. Patients aged $>60$ years are refused RRT and there is preferential allocation to patients without accompanying chronic co-morbidity. Determining the most ethical allocation requires considerations of justice and equity. Moosa and Kidd draw attention to the dangers and injustice inherent in such a rationing of this scarce resource. ${ }^{6}$

Before deciding to commence HIV-positive-to-positive transplantation at GSH, HIV-infected patients with ESRF were believed to be poor transplant candidates and were not accepted for RRT. Moreover, until 2004, HIV-infected patients were deemed unsuitable for renal transplantation owing to the lack of an ART programme, and the dangers of using immunosuppressive antirejection drugs in the absence of HAART. This concern has abated as good outcomes have been confirmed after transplantation in HIVinfected patients receiving HAART. ${ }^{7,8}$

\section{Solid organ transplantation in HIV- infected patients}

Studies from the USA have shown that there is no difference in patient outcome, graft survival and opportunistic infection rates in HIV-infected patients undergoing transplant, compared with HIV-seronegative patients. ${ }^{9-11}$ Many immunosuppressive drugs controlling rejection in transplant patients retard HIV replication; ${ }^{12,13}$ mycophenolate mofetil acts synergistically with some nucleoside analogues, and sirolimus down-regulates the CCR5 co-receptor on CD4+ T-cells, which plays a vital role in entry of HIV into the cell. ${ }^{14,15}$ More rapid immune reconstitution has also been reported in HIVpositive patients treated with cyclosporine (as anti-rejection therapy) and HAART versus HAART alone. ${ }^{16}$

With expanding access to HAART, practice in South Africa now permits allocation of RRT to HIV-infected patients. However, the increasing numbers of patients, the insufficiency of available dialysis slots and the lack of HIV-negative organ donors, severely limits the 
ability to offer RRT (even to HIV-negative patients). ${ }^{6}$ We therefore concluded that transplanting a kidney from an HIV-infected donor (that would otherwise have been discarded) into an HIV-positive recipient represented a viable option, given the potentially large pool of HIV-infected donors (10 - 20\% HIV seroprevalence in potential donors referred to Transplant Co-ordinators in Cape Town and Johannesburg).

However, there are several theoretical concerns to this approach. Firstly, there is the risk that the donor kidney will act as a 'Trojan horse', super-infecting the recipient with a recombinant form of virus or with virus of a different clade. ${ }^{17,18}$ However, as HAART is equally effective in suppressing all clades of HIV, replication of superinfecting HIV should rapidly be suppressed, particularly as it is our practice that the recipient is prescribed a protease inhibitor-based regimen following transplantation. There is a further theoretical risk that the donor will transmit drug-resistant virus. ${ }^{19}$ Although this risk is very small at this stage of the HIV epidemic in SA, we accept that resistance rates will inevitably increase over time, as more patients commence HAART and fail first- and second-line therapy. Future strategies to suppress potentially resistant virus would include the use of donor virus genotyping to inform appropriate ART prescribing. The donor kidney may also act as a 'Trojan horse' for other infectious diseases such as tuberculosis, cytomegalovirus, etc. To obviate this, we measure the urine protein-creatinine ratio and perform a baseline renal biopsy to detect donor renal disease before implantation.

In 2010 we reported our experience with the first 4 kidney transplants from HIV-positive donors to HIV-positive recipients. ${ }^{20}$ Since then, 10 more HIV-positive to HIV-positive renal transplants have been performed (at GSH and the UCT Private Hospital). In all patients, surgery was uncomplicated. Following transplantation, ART was recommenced on day 1 or day 2 and all patients received antithymocyte globulin or thymoglobulin induction therapy and an antirejection regimen consisting of mycophenolate mofetil, tacrolimus and prednisone. All patients are currently 1 - 4 years post-transplant and have undetectable viral loads $(<50$ copies $/ \mathrm{ml})$.
In conclusion, we have shown that transplanting HIV-infected patients with ESRF with kidneys from HIV-positive donors represents a significant advance, benefiting patients with ESRF due to HIVAN, within our resource-constrained context.

1. The National HIV and Syphilis Prevalence Survey, South Africa 2007. National Department of Health, South Africa 2008. http://www.doh.gov.za/docs/reports/2007/antenatal/antenatal_report.pdf (accessed 18 December 2008)

2. Han TM, Naicker S, Ramdial PK, Assounga AG. A cross-sectional study of HIV-seropositive patients with varying degrees of proteinuria in South Africa. Kidney Int 2006;69(12):2243-2250.

3. Winston J, Klotman PE. HIV-associated nephropathy. Mt Sinai J Med 1998:65(1):27-32.

4. Fabian J, Katz T, Gerntholtz T. Chronic kidney disease in HIV infection. Panminerva Med 2007:49(2):51-66.

5. Wools-Kaloustian KK, Gupt SK. Will there be an epidemic of HIV-related chronic kidney disease in Sub-Saharan Africa? Too soon to tell. Kidney Int 2008;74(7):925-929.

6. Moosa MR, Kidd M. The dangers of rationing dialysis treatment: the dilemma facing a developing 6. Moosa MR, Kidd M. The dangers of ration
country. Kidney Int 2006;70:1107-1114.

country. Kidney Int 2006;70:1107-1114.
7. Halpern SD, Asch DA, Shaked A, et al. Determinants of transplant surgeons' willingness to provide 7. Halpern SD, Asch DA, Shaked A, et al. Determinants of transplant surgeons' willingness
organs to patients infected with HBV, HCV or HIV. Am J Transplant 2005;5(6):1319-1325.

organs to patients infected with HBV, HCV or HIV. Am J Transplant 2005;5(6):1319-1325.
8. Pelletier SJ, Norman SP, Christensen LL, et al. Review of transplantation in HIV patients during the HAART era. Clin Transpl 2004:63-82.

9. Roland ME, Barin B, Carlson L, et al. HIV-infected liver and kidney transplant recipients: 1- and 3-year outcomes. Am J Transplant 2008;8(2):355-365.

. Roland ME, Stock PG. Solid organ transplantation is a reality for patients with HIV infection. Curr HIV/AIDS Rep 2006;3(3):132-138.

1. Stock PG, Barin B, Murphy B, et al. Outcomes of kidney transplantation in HIV-infected recipients. N Engl J Med 2010;363(21):2004-2014

12. Frasesetto LA, Browne M, Cheng A, et al. Immunosuppressant pharmacokinetics and dosing modifications in HIV-1 infected liver and kidney transplant recipients. Am J Transplant 2007;7(12):2816-2820

13. Frasetto L, Baluom M, Jacobsen W, et al. Cyclosporin pharmacokinetics and dosing modifications in human immunodeficiency virus-infected liver and kidney transplant recipients. Transplantation in human immun

14. Ciuffreda D, Pantaleo G, Pascuel M. Effects of immunosuppressive drugs on HIV infection: implications for solid-organ transplantation. Transpl Int 2007;20:649-658.

15. Rizzardi GP, Harari A, Capiluppi B, et al. Treatment of primary HIV-1 infection with cyclosporin A coupled with highly active antiretroviral therapy. J Clin Invest 2002;109:681-688.

16. Margolis D, Heredia A, Oldach D, et al. Abacavir and mycophenolic acid, an inhibitor of inosine monophosphate dehydrogenase, have profound and synergystic anti-HIV activity. J Acquir Immune Defic Syndr 1999;21:362-370.

17. Streeck H, Li B, Poon AF, et al. Immune driven recombination and loss of control after HIV superinfection. J Exp Med 2008; 205(8):1789-1796.

18. Van Der Kuyl A, Cornelissen M. Identifying HIV-1 dual infections. Retrovirology 2007,4:67-672.

19. Goulder P, Walker B. HIV-1 superinfection - a word of caution. N Engl J Med 2002;347(10):756-758.

20. Muller E, Kahn D, Mendelson M. Renal transplantation between HIV-positive donors and recipients. N Engl J Med 2010;362(24):2337.

Accepted 12 March 2012. 